\title{
Determination of Detection Limits and Quantitation Limits for Compounds in a Database of GC/MS by FUMI Theory
}

\author{
Shinya Nakashima ${ }^{* 1}$ and Yuzuru Hayashi ${ }^{2}$ \\ ${ }^{1}$ Department of Business Development, Science Software Co., Ltd., 2-8-5 Shinkawa, Chuo-ku, Tokyo 104-0033, Japan \\ ${ }^{2}$ Faculty of Pharmaceutical Sciences, Teikyo Heisei University, 4-21-2 Nakano, Nakano-ku, Tokyo 164-8530, Japan
}

\begin{abstract}
The aim of this paper is to propose a stochastic method for estimating the detection limits (DLs) and quantitation limits (QLs) of compounds registered in a database of a GC/MS system and prove its validity with experiments. The approach described in ISO 11843 Part 7 is adopted here as an estimation means of DL and QL, and the decafluorotriphenylphosphine (DFTPP) tuning and retention time locking are carried out for adjusting the system. Coupled with the data obtained from the system adjustment experiments, the information (noise and signal of chromatograms and calibration curves) stored in the database is used for the stochastic estimation, dispensing with the repetition measurements. Of sixty-six pesticides, the DL values obtained by the ISO method were compared with those from the statistical approach and the correlation between them was observed to be excellent with the correlation coefficient of 0.865 . The accuracy of the method proposed was also examined and concluded to be satisfactory as well. The samples used are commercial products of pesticides mixtures and the uncertainty from sample preparation processes is not taken into account.
\end{abstract}

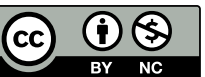

Copyright (c) 2016 Shinya Nakashima and Yuzuru Hayashi. This is an open access article distributed under the terms of Creative Commons Attribution License, which permits use, distribution, and reproduction in any medium, provided the original work is properly cited and is not used for commercial purposes.

Please cite this article as: Mass Spectrom (Tokyo) 2016; 5(1): A0043

Keywords: GC/MS, detection limit, ISO 11843-7, FUMI theory, database

(Received November 10, 2015; Accepted February 9, 2016)

\section{INTRODUCTION}

Gas chromatography/mass spectrometry (GC/MS) has long been the method of choice for identifying and quantifying volatile compounds in complex mixtures and recently, automated and integrated systems have been developed. ${ }^{1-6)}$ The goal of the recent systems is to reduce the human effort made to analyze compounds while maintaining the high level of reliability associated with traditional GC/MS analyses. A sophisticated system, e.g., AMDIS (Automated Mass Spectral Deconvolution \& Identification System), ${ }^{4}$ succeeded in integrating all the necessary procedures for first extracting mass spectra from GC/MS and then comparing each of these spectra with those of target compounds represented in a reference library to identify individual compounds.

The mass spectra contained in a database, if obtained through electron ionization, are highly characteristic and, to a large extent, are instrument-independent. Consequently, the spectra observed in one location can be used elsewhere. On the other hand, the chromatographic characteristics are affected by operating conditions such as the carrier gas flow rate and thus, the retention times and peak areas are instrument-inherent. For analytical purposes, a straightforward approach would have demanded that these precarious quantities be confirmed through experiments with standard samples.

However, Kadokami et al. developed a technique for making the performance of a GC/MS system used as close as possible to that used to construct the database by adjusting the operating conditions with the aid of system check standards (SCSs). ${ }^{1-3)}$ Now, the analysis for identification and quantitation is feasible without standard substances, and yet with a nearly identical accuracy to that with the standards. Its application covers micropollutants, illicit drugs, etc. ${ }^{1-3,7,8)}$

The precision, or rather the uncertainty of measurements, is the major unifying principle of the quality of data. ${ }^{9-11)}$ The procedure to judge the quality such as suitability and capability of analysis is known as validation. Among validation characteristics are the detection limit (DL) and quantitation limit (QL), which show the broad aspect of importance in a variety of fields of analytical chemistry. The DL is usually expressed in terms of standard deviation (SD) or relative standard deviation (RSD) of measurements. However, as is well-known in statistics, the chi-square distribution speci-

* Correspondence to: Shinya Nakashima, Department of Business Development, Science Software Co., Ltd., 2-8-5 Shinkawa, Chuo-ku, Tokyo 104-0033, Japan, e-mail: s-nakashima@science-soft.co.jp 


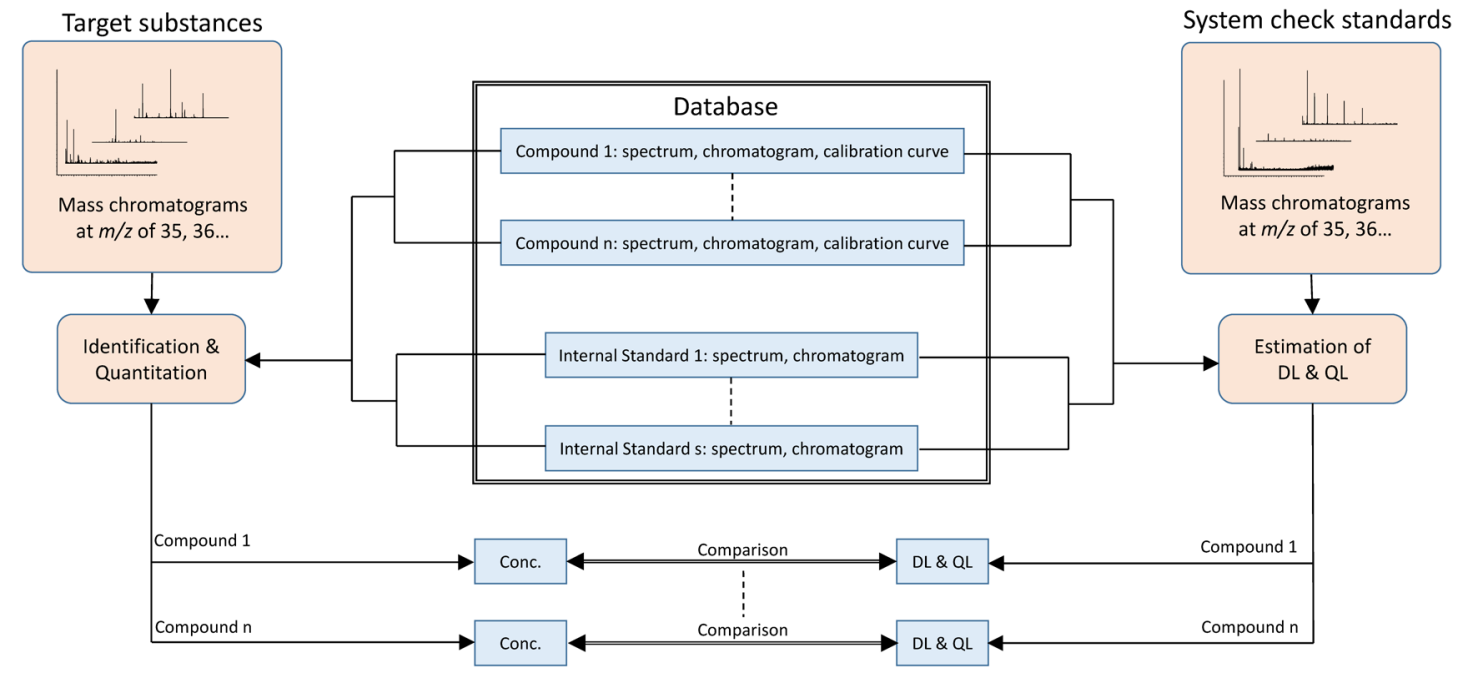

Fig. 1. Integrated GC/MS system for identification, quantitation and validation.

fies the difficulty in obtaining an exact SD estimate from a small number of repeated measurements. For example, the $95 \%$ confidence intervals of an estimated SD are $c a . \pm 20 \%$ of the true value for $n=40$, but $c a$. $\pm 60 \%$ for $n=6$.

The reliability of the former $( \pm 20 \%)$ will be acceptable, but not the number of repetition even in a fast analytical method from the viewpoints of economy and environment, if real samples are consumed. Otherwise, a small number of repetition will be helpful to save time, energy and material at the sacrifice of estimation reliability. However, this would transgress the social purpose of validation, that is, the universal consistency of data.

Of course, the statistical methods are not the only alternatives. This paper proposes a stochastic method for the uncertainty estimation in GC/MS systems. The method, called FUMI theory (FUnction of Mutual Information), ${ }^{12)}$ adopted in ISO 11843 Part $7^{13)}$ enables us to obviate the need for the repetition of measurement while keeping the reliability of the estimation as high. Therefore, it is suitable for handling a large number of analyte data in a database of a GC/MS system. The statistical confidence level of analytical results should not be compromised by the loosened strictness of validation. The GC/MS system here is constructed according to the technique established by Kadokami et al. ${ }^{1)}$ and its validity is proved experimentally with sixty-six pesticide samples.

\section{EXPERIMENTAL}

\section{Reagents}

Acetone was of pesticide residue/polychlorinated biphenyl analysis grade (Wako Pure Chemical Industries, Ltd., Osaka, Japan). Test samples for DL and QL were 69 Pesticides Mix (Part No. 99056008, Hayashi Pure Chemical; substance names are listed in the legend of Fig. 5). Internal standard solutions $\left(500 \mathrm{mg} / \mathrm{L}\right.$ of 1,4 -dichlorobenzene- $d_{4}$, naphthalene- $d_{8}$, acenaphthene- $d_{10}$, phenanthrene- $d_{10}$, fluoranthene- $d_{10}$ and chrysene- $d_{12}$ ) were obtained from Hayashi Pure Chemical. Test sample solutions for determination were prepared by adding aliquot volumes of the internal standard solutions to $1 \mathrm{~mL}$ acetone solutions containing designated amounts of all the target chemicals $(0.01,0.05,0.10$, 0.20 or $0.50 \mathrm{mg} / \mathrm{L}$ ).
SCSs were Naginata Criteria Samples Mix II (Part No. 99056015, Hayashi Pure Chemicals) containing the aforementioned internal standards, captafol, isoxathion, 2,4-dichloroaniline, 2,4-dinitroaniline, pentachlorophenol, simazine, fenitrothion, and decafluorotriphenylphosphine (DFTPP) at $1 \mathrm{mg} / \mathrm{L}$ each. The DFTPP tuning and retention time locking were carried out as described elsewhere. ${ }^{2,14)}$

\section{Instruments and software}

An Agilent 7890B/5975C GC/MS system with an HP$5 \mathrm{~ms}$ capillary column ( $30 \mathrm{~m}$ length, $0.25 \mathrm{~mm}$ i.d., $0.25 \mu \mathrm{m}$ Film Thickness. Agilent Technologies, CA, USA) was used under the conditions that have already been reported. ${ }^{2)}$ The software used for identification and quantification was NAGINATA $^{\text {TM }}$ version 1.00.03 (Nishikawa Keisoku Co., Ltd., Tokyo, Japan). The ISO $11843-7^{13)}$ method for DL and QL was conducted with a software called TOCO version 2.0 (Institute of FUMI Theory, Chiba, Japan); 512 data points of observed baselines were handled for this purpose.

\section{Theoretical features of the integrated system pro- posed}

Figure 1 illustrates the overview of the integrated system proposed here for identification, quantitation and validation. Like ordinary GC/MS systems, the database stores spectra, chromatograms and calibration curves of registered compounds. The GC inlet, column and tuning conditions of user's GC/MS system need be adjusted with SCSs to make the ready-made database applicable to user's system. ${ }^{1-3)}$ The criterion for the adjustments carried out here has been validated so far. ${ }^{1)}$ After these steps, the newly constructed GC/ MS system is ready for identification and quantitation of real samples (see the left side of Fig. 1).

In usual GC/MS analyses, the SCSs are not used for any other purposes than the above system adjustments. However, the validation proposed here demands further utilization of the SCSs. The analytical information (see below) to be elicited from them, with the aid to the elements of the database, leads to the DL and QL estimates for the chemical substance registered there. It should be noted that this uncertainty estimation for validation (the right side of Fig. 1) can be conducted separately from measuring target samples (the left side). 


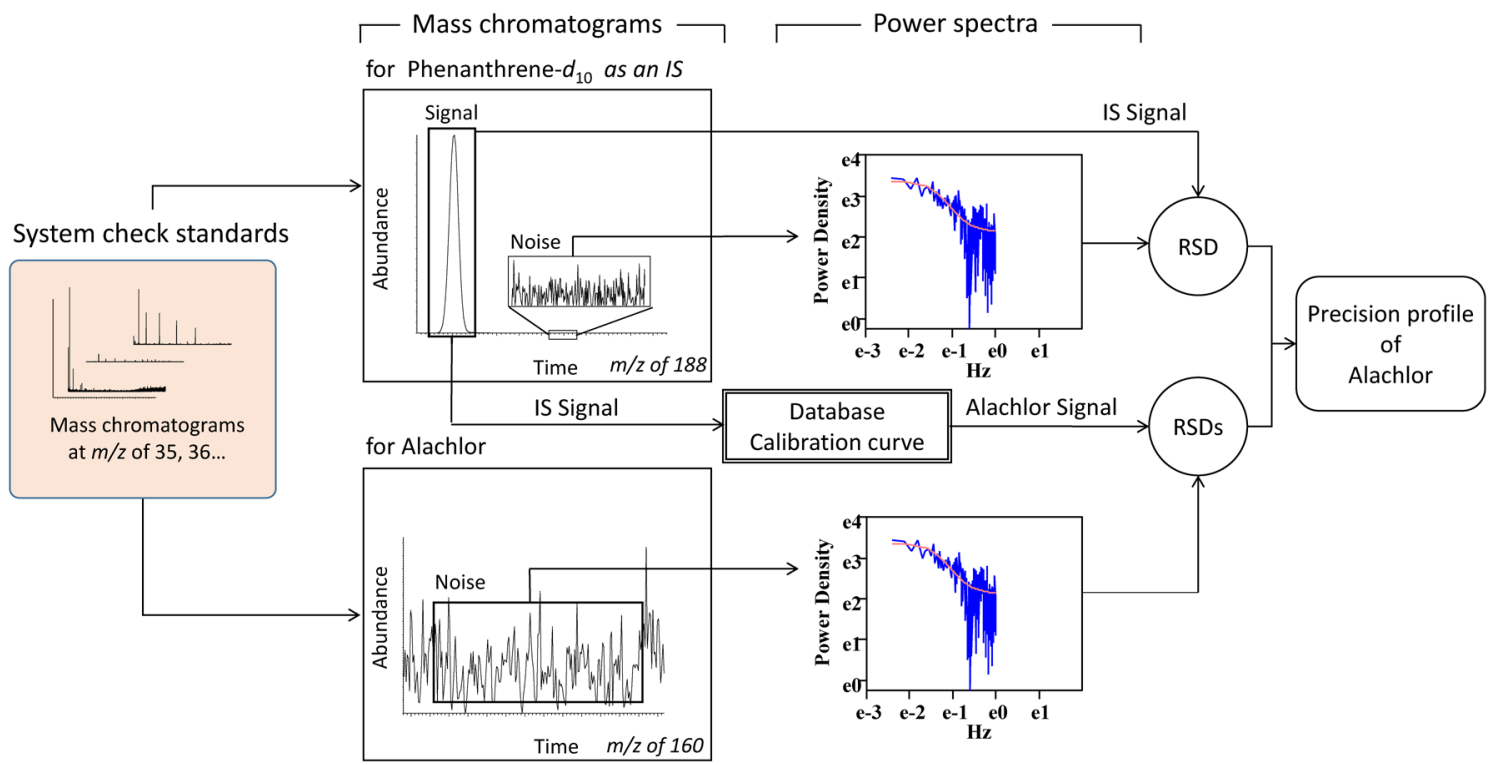

Fig. 2. Estimation of detection limit and quantitation limit of alachlor with phenanthrene- $d_{10}$ as an internal standard.

The zigzag lines of the power spectra denote the observed noise power spectra and the smooth lines the least squares fitted lines for phenanthrene- $d_{10}$ (upper) and alachlor (lower), respectively.

According to ISO 11843 Part 7, the DL and QL estimates can directly be obtained through a precision profile which is defined to be the RSD of measurements as a function of sample concentration. ${ }^{13,15)}$ For the internal standard (IS) method, the $Y$-axis of the precision profile is the RSD of the ratio, $R$, of target area measurement, $A_{\mathrm{T}}$, to IS area measurement, $A_{\mathrm{I}}\left(R=A_{\mathrm{T}} / A_{\mathrm{I}}\right)$. The theory of the propagation of indeterminate errors ${ }^{16)}$ tells that the relative variance squared of the above ratio takes the form ${ }^{17)}$ :

$$
\left(\frac{s_{\mathrm{R}}}{\bar{R}}\right)^{2}=\left(\frac{s_{\mathrm{I}}}{\overline{A_{\mathrm{I}}}}\right)^{2}+\left(\frac{s_{\mathrm{T}}}{\overline{A_{\mathrm{T}}}}\right)^{2}
$$

where $s_{\mathrm{R}}$ denotes the $\mathrm{SD}$ of measured ratios, $R\left(=A_{\mathrm{T}} / A_{\mathrm{I}}\right) ; s_{\mathrm{I}}$ the SD of IS measurements, $A_{\mathrm{I}} ; s_{\mathrm{T}}$ the SD of target measurements, $A_{\mathrm{T}} ; \bar{Z}$ the mean of random variable $Z$. Hereafter, Eq. (1) is referred to as precision profile.

First, we estimate the term of the right side of Eq. (1) from experimental data of SCSs. The following factors are inevitable for creating the precision profile (Eq. (1)):

A: time variation in background noise for $s_{\mathrm{I}}$ and $s_{\mathrm{T}}$; B: signal areas, $A_{\mathrm{I}}$ and $A_{\mathrm{T}}$; C: signal widths for $s_{\mathrm{I}}$ and $s_{\mathrm{T}}$;

(for details, see the following section). All these factors need to be determined for each of user's systems, mainly because mathematical properties of noise and signal are different from system to system.

The upper half of Fig. 2 shows the procedures to calculate the $\operatorname{RSD}\left(=s_{\mathrm{I}} / A_{\mathrm{I}}\right)$ for an IS (here, phenanthrene- $\left.d_{10}\right)$ from the data of the SCSs. Similarly as for a target compound (here, alachlor; see the lower part of the figure), a part of the baseline of an ion chromatogram at the same $\mathrm{m} / \mathrm{z}$ as that of the target can be the desired time variation (factor $\mathrm{A}$ ). However, factors $\mathrm{B}$ and $\mathrm{C}$ for the target are problematic, because neither of them can be found in the SCSs data.

We can calculate factor B through the linear calibration curve of the target compound which is stored in the database:

$$
Y=a X
$$

where $Y, a$, and $X$ denote, respectively, the area measurement ratio of target to IS, the slope and the concentration of the target. Let $A_{\mathrm{I}}^{*}$ be the area of the IS peak provided by the SCSs (actually $\left.A_{\mathrm{I}}^{*}=A_{\mathrm{I}}\right)$. Noticing that $Y$ is the ratio $\left(=A_{\mathrm{T}} / A_{\mathrm{I}}^{*}\right)$, we can describe the area estimate of the target compound which is inherent to the user's apparatus:

$$
A_{\mathrm{T}}=A_{\mathrm{I}}^{*} a X
$$

as a function of $X$. In Fig. 2, the transformation by Eq. (3) is designated by the block of "database calibration curve."

Factor $\mathrm{C}$ can be determined with referring to the signal in an ion chromatogram registered in the database under the assumption that peak width would not substantially be different from the original system for database creation if the appropriate adjustment with the SCSs is performed in the user's system.

Because the concentration of the IS is often fixed in practice, the precision profile (Eq. (1)) can be a function of sample concentration, $X$.

\section{AND QL ESTIMATION BY ISO 11843 PART 7}

The FUMI theory is a theory to estimate the SD of measurements from stochastic properties of noise as well as signal in the output of analytical instruments such as chromatographs and mass spectrometers. ${ }^{12)}$ Instrumental prerequisites of the theory are: the background noise can well be approximated by a mixed random processes of the white noise and Markov process alone; the background noise is a predominant source of measurement uncertainty. The FUMI theory has successfully been applied to a lot of analytical instruments. ${ }^{12,18,19)}$

Factors, $\mathrm{A}$ and $\mathrm{C}$, mentioned above are required by this theoretical SD estimation. The SD, $s_{\mathrm{I}}$ and $s_{\mathrm{T}}$ in Eq. (1), can be estimated by mathematical techniques mainly made up of the Fourier transform of the noise (factor C) into a power spectrum and the nonlinear least squares fitting of the model random process of the white noise and Markov process to 
the observed power spectrum.

Under the assumption that the SD of concentration estimates, $s_{\mathrm{X}}$, is constant, often called homoscedastic situation, the detection limit, $x_{\mathrm{d}}$, is defined as ${ }^{13,15)}$

$$
x_{\mathrm{d}}=\left(k_{\mathrm{c}}+k_{\mathrm{d}}\right) s_{\mathrm{X}}
$$

where $k_{c}$ and $k_{\mathrm{d}}$ denote the coefficients to specify the probabilities of an error of the first kind and second kind, respectively. If the concentration estimates are normally distributed, and $k_{\mathrm{c}}=k_{\mathrm{d}}=1.65$, Eq. (4) takes the simple form that $s_{\mathrm{X}} / x_{\mathrm{d}}=1 / 3.3 \approx 30 \%$. Practically, if the calibration curve is a straight line passing through the origin, the detection limit is equal to the concentration at which the RSD of measurements (the left side of Eq. (1)) takes $30 \%$ in the precision profile. $^{13,15)}$

Similarly, the quantitation limit can be interpreted as the concentration at which the RSD is $10 \%^{20)}$ in the precision profile (here, $k_{\mathrm{c}}+k_{\mathrm{d}}=10$ ).

\section{RESULTS AND DISCUSSION}

A noise power spectrum is produced through the Fourier transform of the background noise of a chromatogram. Figure 2 illustrates the power spectra (zigzag lines) of an IS, phenanthlene- $d_{10}$, and target compound, alachlor. The smooth lines of the figures denote the respective simplex least squares fitting of the theoretical power spectra to the observed lines. The goodness of fit of the least-squares is a useful indicator for the successful application of the FUMI theory. In Fig. 2, the fitting goodness looks satisfactory. The least-squares fitting is performed not on the logarithmic scale but on the linear scale and then, the quality of fit might seem to be poor around and above $0.5 \mathrm{~Hz}$. In practice, the least-squares fitting can provide three parameters that are indispensable for describing background noise in the theory: one for the white noise and two for the Markov process.

Through the above procedures for the fitting as shown in Fig. 2 and according to Eq. (1), we can obtain the precision profile for alachlor (see Fig. 3). The theoretical prediction (-) can be considered in good agreement with the experimental results from repetition $(\bullet ; n=6)$, since the former line falls within the $95 \%$ confidence intervals of the RSD estimates. This result corroborates, besides the usefulness of the FUMI theory, the generally-accepted concept that the internal standard method is helpful to compensate injection errors in analytical instruments.
The graphical estimation of DL and QL, described in ISO 11843-7, is also demonstrated in Fig. 3 (see the arrows). The estimated values of DL and QL for alachlor are: ISO-based $0.020 \mathrm{mg} / \mathrm{L}$ and $0.067 \mathrm{mg} / \mathrm{L}$, respectively; statistically $(n=6)$, $0.022 \mathrm{mg} / \mathrm{L}$ and $0.074 \mathrm{mg} / \mathrm{L}$, respectively (for both, Eq. (4) is used). The ISO method is very simple and useful, and its reliability in GC/MS systems will be verified hereafter.

Figure 4 shows the ion chromatograms of alachlor at concentrations around the estimated DL $(=0.020 \mathrm{mg} / \mathrm{L})$ : (A) $0.01 \mathrm{mg} / \mathrm{L}$; (B) $0.05 \mathrm{mg} / \mathrm{L}$. The DL signal is twice the signal of the upper figure in area. Its signal-to-noise ratio was observed to be about 4.0. This $S / N$ might appeal to the practical sense of chromatographers. However, as far as precision or uncertainty is concerned, noise is often elusive due to autocorrelation and the $S / N$ is not always an honest yardstick. ${ }^{18,21)}$

Figure 5 shows the correlation between the DL estimates by the FUMI theory and those by the repeated measurements $(n=6)$. The differently estimated values for 66 pesticides are strongly correlated $(r=0.865)$. For example, as for alachlor, the DL value estimated by the FUMI theory $(0.020 \mathrm{mg} / \mathrm{L})$ is close to that by the repetition experiments $(=0.022 \mathrm{mg} / \mathrm{L})$.

Sixty-six pesticides were selected for this study out of 69 chemicals of 69 Pesticides Mix. Two chemicals were excluded because of no entry in the NAGINATA ${ }^{\mathrm{TM}}$ database (trichlorfon and CNP amino type) and one because of appearances of troublesome multi isomer peaks (propiconazol). For 55 pesticides out of 66 , the SD values were

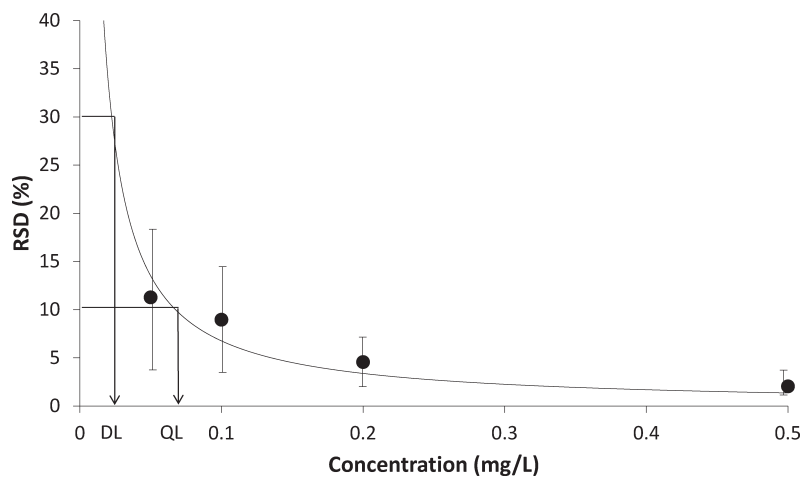

Fig. 3. The precision profile of alachlor with phenanthrene- $d_{10}$ as IS. The bars denote the $95 \%$ confidence intervals of the estimates $(n=6)$.

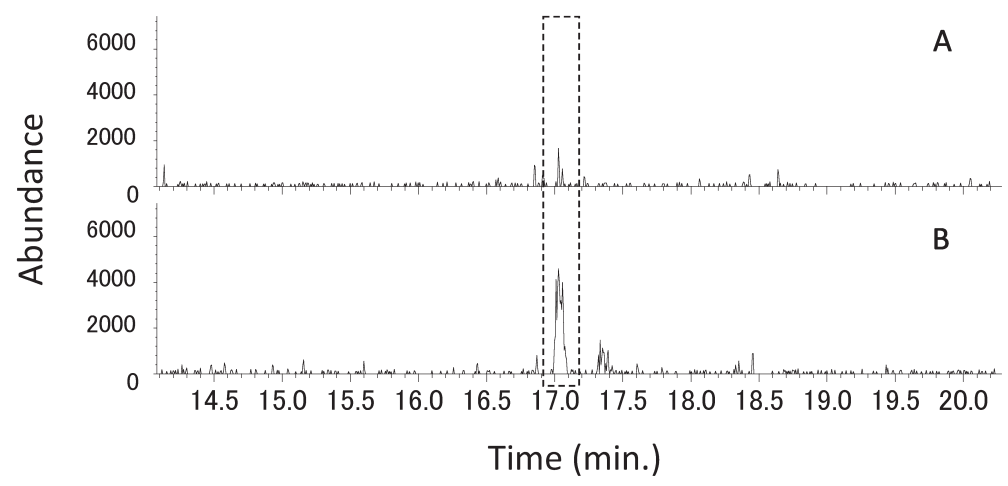

Fig. 4. Observed signals of alachlor around the detection limit. Sample concentrations are (A): $0.01 \mathrm{mg} / \mathrm{L} ;(B): 0.05 \mathrm{mg} / \mathrm{L}$ 


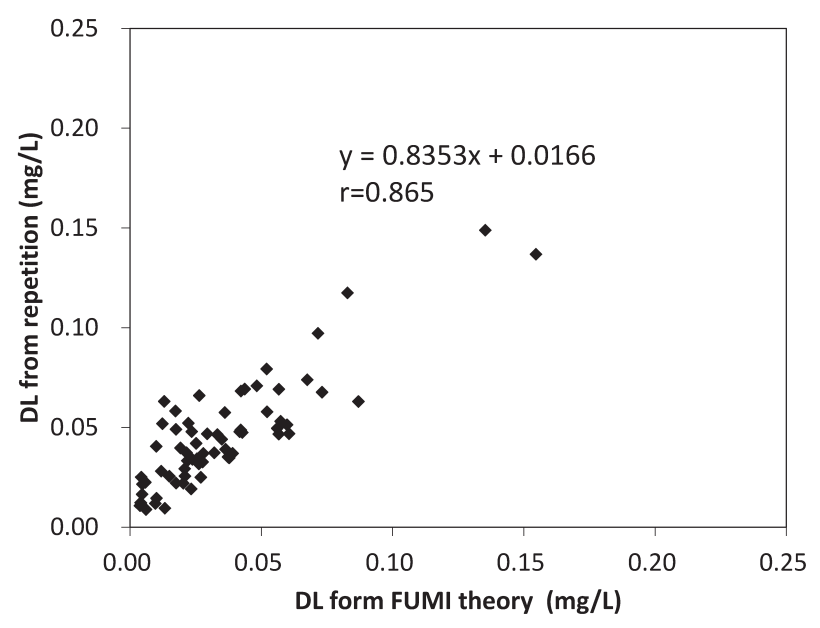

Fig. 5. Correlation between the FUMI theory and repetition.

The statistical DL values were obtained at a concentration of $0.5 \mathrm{mg} / \mathrm{L}$ for $\alpha$-endosulfan, alachlor, atrazine, $\beta$-endosulfan, benfluralin, bifenox, bromobutide, buprofezin, butamifos, cafenstrole, captan, chlornitrofen (CNP), chloroneb, chlorothalonil (TPN), chlorpyrifos, diazinon, dichlorvos (DDVP), dimepiperate, dimethametryn, dimethoate, disulfoton, dithiopyr, edifenphos, EPN, esprocarb, fenitrothion, fenobucarb, fenthion, fthalide, iprobenfos, iprodione, isofenphos, isoprocarb, isoprothiolane, isoxathion, malathion, mefenacet, metalaxyl, methidathion, methyl dymron, molinate, napropamide, pendimethalin, phenthoate, pretilachlor, procymidone, pyributicarb, pyridaphenthion, pyroquilon, simazine, simetryn, terbucarb, thenylchlor, thiobencarb, tolclofosmethyl, but at $0.1 \mathrm{mg} / \mathrm{L}$ for trifluralin, anilofos, pencycuron, pyriproxyfen, mepronil, propyzamide, flutolanil, etridiazole (echlomezol), ethofenprox, dichlobenil, piperophos at $0.1 \mathrm{mg} / \mathrm{L}$.

estimated from the data at $0.5 \mathrm{mg} / \mathrm{L}$, but for 11 pesticides at $0.1 \mathrm{mg} / \mathrm{L}$. As far as the measurement uncertainty in the GC/ MS system used here is concerned, the noise predominance over the other error sources seemed to be retained at as high a concentration as $0.5 \mathrm{mg} / \mathrm{L}$ for 55 compounds. For the other eleven compounds, however, the $S / N$ values of target peaks were so high that the above noise predominance was ruled out. In the latter case, however, the data at a lower concentration $(0.1 \mathrm{mg} / \mathrm{L})$ yield the excellent results as shown in Fig. 5. Underestimation of uncertainty would ensue from a manipulation of the eleven compounds at the inappropriate high concentration.

Precision and accuracy are both of critical importance in estimating the DL and QL in any analytical instrument. In Fig. 6, the accuracy of our system is demonstrated as the averaged concentration estimates $(n=6)$. All the concentration estimates are included within $\pm 20 \%$ of the actual concentration $(=0.50 \mathrm{mg} / \mathrm{L})$. No bias of the system is observed. The accuracy of the quantification results is comparable to that previously reported with the similar GC/MS system $(n=1)$ : out of 60 compounds, 36 were detected at concentrations within $\pm 30 \%$ of the true value and 58 fell within $\pm 50 \%$ of the true value. $^{3)}$

The principle of the proposed system is illustrated in Figs. 1 and 2 , and its analytical reliability is demonstrated by the statistics shown in Figs. 5 and 6. In the current system, the DL and QL can be determined for each target substance in less than a couple of minutes through the software. The

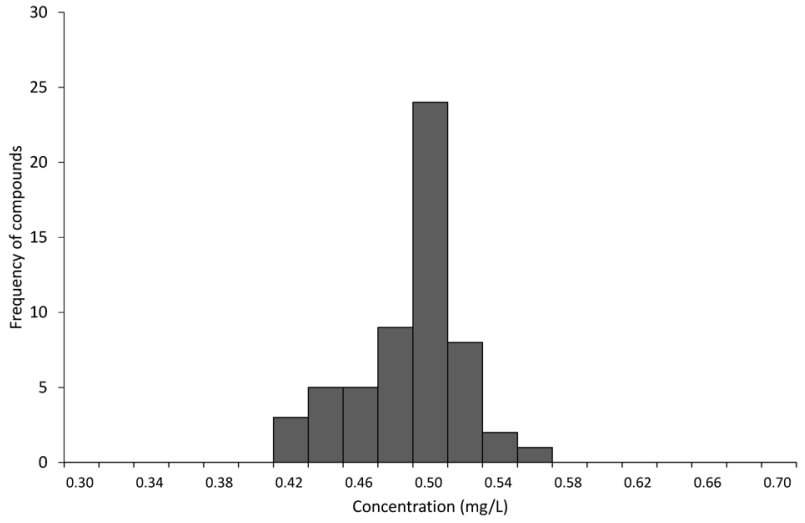

Fig. 6. Histogram of quantification results of $0.50 \mathrm{mg} / \mathrm{L}$ samples $(n=6)$.

Mean $=0.479 \mathrm{mg} / \mathrm{L}, \mathrm{SD}=0.030$.

proposed system could be practical, as long as the number of target substances to be analyzed at the same time is not so large.

It, however, has difficulty in simultaneously handling all compounds registered in a database of a GC/MS system. A time to be spent on the FUMI-theoretical analysis of a chromatogram is not long ( $<2 \mathrm{~min})$, including the manual selections of noise and signal and the run of the software called TOCO (see the Experimental section). However, it would take an enormous amount of time to conduct the above analysis on each of chromatograms in the database. Therefore, developing a new method to solve this problem would be an interesting subject of future study.

Recently, a theoretical paper which uses an auto-correlation function instead of the Fourier transform in ISO 11843-7 for the estimation of noise parameters has been reported. ${ }^{22}$ Procedures for the parametrization are quite simple in the new theory. Furthermore, the number of data necessary for the parametrization is no longer limited to 2 to the $n$-th power as it usually is when the fast Fourier transform algorithm is applied. This theory would possibly be a clue used to find another method for determining the detection limits and quantitation limits simultaneously for over a thousand compounds in a database of a GC/MS system.

\section{Acknowledgements}

We have collected a lot of data in Nishikawa Keisoku Co., Ltd. We would like to take this opportunity to show our gratitude to the company.

\section{REFERENCES}

1) K. Kadokami, K. Tanada, K. Tanabe, K. Nakagawa. Novel gas chromatography-mass spectrometry database for automatic identification and quantification of micropollutants. J. Chromatogr. A 1089: 219-226, 2005.

2) T. Ishida, K. Kudo, S. Naka, K. Toubou, T. Noguchi, N. Ikeda. Rapid diagnosis of drug intoxication using novel NAGINATA $^{\mathrm{TM}}$ gas chromatography/mass spectrometry software. Rapid Commun. Mass Spectrom. 21: 3129-3138, 2007.

3) S. Nakashima, H. JI, T. Yamagami, K. Asai, K. Kadokami, M. Mutsuga, Y. Kawamura, R. Shinohara, K. Arizono. Development of a novel GC/MS database for the determination of additives for 
food packaging into the processed foods. Jpn. J. Food Chem. 20: $42-51,2013$.

4) W. G. Mallard, J. Reed. in Automated Mass Spectral Deconvolution and Identification System AMDIS-USER GUIDE, US Department of Commerce Technology Administration, National Institute of Standards and Technology (NIST), Gaithersburg, MD20899, 1998, pp. 1-2.

5) T. Kind, G. Wohlgemuth, D. Y. Lee, Y. Lu, M. Palazoglu, S. Shahbaz, O. Fiehn. FiehnLib: Mass spectral and retention index libraries for metabolomics based on quadrupole and time-offlight gas chromatography/mass spectrometry. Anal. Chem. 81: 10038-10048, 2009.

6) A. Robbat, A. Hoffmann, K. M. Namara, Y. Huang. Quantitative identification of pesticides as target compounds and unknowns by spectral deconvolution of gas chromatographic/mass spectrometric data. J. AOAC Int. 91: 1467-1477, 2008.

7) K. Kadokami, K. Tanada, K. Taneda, K. Nakagawa. Development of a novel GC/MS database for simultaneous determination of hazardous chemicals. Bunseki Kagaku 53: 581-588, 2004.

8) D. Jinya, T. Iwamura, K. Kadokami. Comprehensive analytical method for semi-volatile organic compounds in water samples by combination of disk-type solid-phase extraction and gas chromatography-mass spectrometry database system. Anal. Sci. 29: 483-486, 2013

9) Analytical Methods Committee. Uncertainty of measurement: Implications of its use in analytical science. Analyst (Lond.) 120: 2303-2308, 1995.

10) Guide to the Expression in Measurement, International Organization for Standardization, 1993.

11) ICH Topic Q2B, 1996.
12) Y. Hayashi, R. Matsuda. Deductive prediction of measurement precision from signal and noise in liquid chromatography. Anal. Chem. 66: 2874-2881, 1994.

13) ISO 11843-7, Capability of detection- Part 7: Methodology based on stochastic properties of instrumental noise, 2012.

14) Method 625, Methods for Organic Chemical Analysis of Municipal and Industrial Wastewater, Base/neutrals and acids, US Environmental Protection Agency, 1995.

15) ISO 11843-5, Capability of detection- Part 5: Methodology in the linear and non-linear calibration cases, 2008.

16) R. A. Day, A. L. Underwood. in Quantitative Analysis, Prentice Hall, Englewood Cliffs, 1988, pp. 548-549.

17) Y. Hayashi, R. Matsuda. Stochastic utility of internal standard method in liquid chromatography. Anal. Sci. 11:389-400, 1995.

18) R. Matsuda, Y. Hayashi, K. Sasaki, Y. Saito, K. Iwaki, H. Harakawa, M. Satoh, Y. Ishizuki, T. Kato. Deductive prediction of precision in measurement, calibration, and standard addition method in atomic absorption spectrometry for cadmium. Anal. Chem. 70: 319-327, 1998.

19) A. Kotani, Y. Yuan, B. Yang, Y. Hayashi, R. Matsuda, F. Kusu. Selection of the optimal solvent grade for the mobile phase in HPLC with electrochemical detection based on FUMI theory. Anal. Sci. 25: 925-929, 2009.

20) The Japanese Pharmacopoeia, 16th Ed., 2012, pp. 1647-1650.

21) J. D. Ingle Jr., R. S. Crouch. in Spectrochemical Analysis, Prentice Hall, NJ (USA), 1988.

22) Y. Hayashi, N. F. Zhang. Evaluation of measurement precision from stationary baseline noise in instrumental analyses. Anal. Sci. 31: 1219-1224, 2015. 